\title{
Neural crest cell migration of mouse B16-F1 melanoma cells transplanted into the chick embryo is inhibited by the BMP-antagonist noggin
}

\author{
CHRISTIAN BUSCH, ULRICH DREWS, CLAUS GARBE, STEFAN R. EISELE and MATTHIAS OPPITZ ${ }^{1}$
}

Section of Dermatologic Oncology, Department of Dermatology, University of Tübingen, Germany

Received June 11, 2007; Accepted July 31, 2007

\begin{abstract}
Melanoma cells are derived from the neural crest and characterized by high migratory potential and invasive growth. To test the analogies between malignant and embryonic cell migration, in previous studies we transplanted melanoma cells and non-transformed mouse neural stem cells into the neural crest compartment of the chick embryo. Human and mouse melanoma cells spontaneously migrated along the neural crest pathways while emigration of neural stem cells was dependent on pre-treatment with BMP-2 (bone morphogenetic protein-2). In the embryo neural crest cell migration is induced by BMP and inhibited by its antagonist noggin. We tested whether the spontaneous neural crest cell migration of melanoma cells was dependent on their endogenously expressed BMP and could be inhibited by noggin. Mouse B16-F1 melanoma cells transfected with GFP-VASP (vasodilatorstimulated phosphoprotein) were cultured as aggregates and treated with BMP-2 or noggin. Untreated and treated aggregates were transplanted into the neural tube of the E2 chick embryo. Untreated and BMP-2-treated melanoma cells emigrated from the neural tube along with the chick host neural crest cells. Noggin-treated aggregates showed no emigration. We conclude that spontaneous emigration of melanoma cells depends on their constitutive overexpression of BMP, and that noggin efficiently suppresses the emigration of melanoma cells in the embryonic micro-environment, thus rendering noggin a promising agent for the inhibition of melanoma cell migration in vivo.
\end{abstract}

\section{Introduction}

Invasive growth of cancer cells has many similarities to physiological migration of the respective precursor cells in the embryo. Therefore it has been proposed that the genes

Correspondence to: Dr Matthias Oppitz, ${ }^{1}$ Present address: Department of Pathology, LKH Feldkirch, A-6800 Feldkirch, Austria

E-mail: matthias.oppitz@1khf.at

Key words: melanoma, bone morphogenetic protein-2, noggin, chick embryo, neural crest, B16-F1, cell migration responsible for the migratory potential of embryonic cells which are silenced in the course of differentiation are reactivated during malignant transformation (1-3). We have developed a novel experimental model to test this hypothesis by transplanting melanoma cells back into the embryonic environment of their precursor cells $(4,5)$.

Melanocytes are derived from the embryonic neural crest. After closure of the neural tube in the trunk of the embryo, neural crest cells migrate along the medial and lateral pathways. Neural crest cells following the medial pathway form spinal ganglia and the autonomic ganglia of the sympathetic chain. The melanocyte precursors follow the lateral pathway (6). After neural crest cell migration along the lateral pathway, the melanoblasts colonize the epidermis and are the source of melanocytes in the adult individual. Melanomas arise from melanoblasts or melanocytes in the epidermis and are characterized by a high migratory potential and invasive growth.

In previous studies we observed that after transplantation of human SK-Mel 28 (4) and mouse B16-F1 (5) melanoma cells into the neural tube of the early chick embryo the melanoma cells integrated into the neural crest and participated in neural crest cell migration. In paraffin sections, the transplanted melanoma cells were identified with HMB-45 antibody and with human and mouse specific DNA in situ hybridization. HMB-45 is directed against premelanosomes and is used as a specific marker for the melanocytic lineage (7). In addition, neural crest-specific HNK-1 antibody was applied on whole mount embryos and paraffin sections to compare migrating melanoma cells to host neural crest cells. Melanoma cells (HNK-1-negative) had emigrated along the medial and the lateral neural crest cell pathways and could be detected laterally in the prospective epidermis and medially in the para-aortic sympathetic ganglia among HNK-1-positive host neural crest cells (5).

In the experiments with mouse melanoma cells we used the GFP-transfected B16-F1 melanoma cell line which allowed the monitoring of active migration with live fluorescence. We observed segmental fluorescence patterns of melanoma cells actively migrating along the medial and lateral neural crest cell pathways corresponding to autochthonous neural crest cell migration.

The mouse B16 melanoma cell line was established by Kinsey (8). The subline B16-F1 is characterized by a high metastatic potential $(9,10)$. Transfection with the VASP 
(vasodilator-stimulated phosphoprotein)-GFP construct (11) was performed by Rottner (12). VASP-GFP expression is linked to adhesion sites with the adaptor proteins vinculin and zyxin. High fluorescence is located in the anterior tips of lamellipodia and the level of GFP-VASP recruitment is proportional to the protrusion rate, thus indicating active cell migration (12).

In contrast to the transformed melanoma cells, nontransformed neural stem cells depend on BMP-2 (bone morphogenetic protein-2) treatment to perform neural crest cell migration in the same experimental conditions (13). When neurospheres generated from adult mouse subventricular zone (SVZ) stem cells were treated with BMP-2 for $24 \mathrm{~h}$ and transplanted into the neural tube of the chick embryo, only BMP-2 pre-treated neurospheres integrated into the neural crest and performed neural crest cell-like migration. This is in line with the observation that in vitro adult neural stem cells from the SVZ can be induced to differentiate into neural crest cell fates by BMP-2 treatment (14). On the basis of these experiments we supposed that integration of melanoma cells into the neural crest was also BMP dependent. This study was designed to test this hypothesis.

In the chick embryo, BMP-2 and -4 are expressed in the dorsal neural tube between stages 12 to 20 according to Hamburger and Hamilton (15). BMPs induce neural crest formation and migration of neural crest cells (16). BMPs are members of the transforming growth factor- $\beta$ (TGF- $\beta$ ) superfamily. They signal through serine/threonine kinase receptors designated as type I (BMPR-IA and -IB) and type II (BMPR-II) (17). BMP-2, -4 , and -7 are constitutively overexpressed in malignant melanoma cells in vitro (18). Because of the endogenous overexpression of BMPs in melanoma cells we used the BMP-antagonist noggin to analyze the BMP-effect. Noggin is a soluble molecule that antagonizes the actions of BMP-2 (19), BMP-4 (20), and BMP-7 $(21,22)$ with a marked preference for BMP-2 and -4 over BMP-7 (22). Noggin is expressed in the neural tube and in the notochord and disappears in the prospective neural crest area (15). By binding tightly to BMPs, noggin prevents them from binding to their receptors (20).

In contrast to our previous studies in which melanoma cell suspensions were used, in the present experiment B16-F1 cell aggregates were transplanted. Aggregates, which were similar to neurospheres, were prepared in rotation culture. During the 24-h rotation culture, melanoma cell aggregates were treated with BMP-2 or noggin. After transplantation into the neural tube of the 2-day chick embryo, integration into the neural crest and the patterns of emigration were compared between treated and untreated aggregates by epifluorescence in the live embryos and by immunohistochemistry with HMB-45 in paraffin serial sections of the same embryo.

\section{Materials and methods}

Cell line and culture. B16-F1 cells were cultivated with RPMI-medium (RPMI-1640, Biochrom, Berlin, Germany), supplemented with $10 \%$ fetal calf serum (Biochrom), $2 \mathrm{mM}$ L-Glutamine, $100 \mathrm{U} / \mathrm{ml}$ Pen-Strep (Roche, Mannheim, Germany) and $25 \mathrm{mM}$ Hepes-buffer (Biochrom). Cells were proliferated for 3 days until confluency. B16-F1 melanoma cells were used at passages 30-35. Twenty-four hours prior to transplantation, B16-F1 cells were trypsinized ( $0.1 \%$ trypsin) and cell concentration was adjusted to $10^{6}$ cells $/ \mathrm{ml}$.

Generation of melanoma cell aggregates. The cell suspension $(1 \mathrm{ml})$ was transferred into sterile gas permeable biofoil bags [Biofoil, Heraeus-Kulzer, Hanau, Germany (23)]. Before sealing, $20 \mathrm{ng} / \mathrm{ml} \mathrm{BMP-2}$ or $100 \mathrm{ng} / \mathrm{ml}$ noggin (both from R\&D Systems, Wiesbaden, Germany) were added. Bags were sealed with an electrical sealing device after filling until there was no air. Bags were placed in open cylindrical plastic containers and were continuously kept in constant double rotation $(2 \mathrm{rpm})$ in a humidified cell incubator at $37^{\circ} \mathrm{C}$ with $95 \%$ air and $5 \% \mathrm{CO}_{2}$.

Transplantation into the chick embryo. Fertilized eggs of White Leghorn Chickens (Gallus Gallus domesticus) were obtained from a hatchery (Weiss, Kilchberg, Iller, Germany) and incubated at $37.5^{\circ} \mathrm{C}$ in a temperature-controlled brooder (Ehret, Emmendingen, Germany). The eggs were fenestrated after $48 \mathrm{~h}$ of incubation [equal to stage 12-13 according to Hamburger and Hamilton (24)]. One B16-F1 aggregate or a part thereof was transplanted into the neural tube using Kwik-Fil $^{\text {TM }}$ Borosilicate Glass Capillaries and a mouth pipette (World Precision Instruments, Inc., Sarasota, FL) under a Zeiss stereo microscope. The technique of transplantation with a calibrated micro-pipette requires a specific aggregate size which cannot be surpassed. Therefore, the transplant in each embryo was of comparable size. This was confirmed by microscopical documentation directly after the transplantation in each case. For documentation of the transplantation, cell aggregates were stained with nile blue sulphate (Bayer, Leverkusen, Germany). After transplantation, the aggregate remained at the site of transplantation, and was documented. Eggs were sealed with adhesive tape (Super88, 3 M, St. Paul, $\mathrm{MN}$ ), and kept in the brooder for 24 or $48 \mathrm{~h}$.

Histology and immunohistochemistry. Chick embryos were fixed in $4 \%$ buffered paraformaldehyde for 2-4 h, depending on the size of the embryo. After rinsing with PBS, samples were dehydrated with ethanol, treated with xylene, and embedded in paraplast. Serial sections of $5 \mu \mathrm{m}$ were mounted on polylysin-coated slides. Every 10th section was stained with H\&E. After deparaffinization and rehydration, tissues were treated with Proteinase $\mathrm{K}$ (Sigma-Aldrich), $20 \mu \mathrm{g} / \mathrm{ml}$ in TRIS- $\mathrm{HCl}$, at $37^{\circ} \mathrm{C}$ for $30 \mathrm{~min}$. Then, HMB-45 monoclonal antibody preparation (1:20, Dako, Germany), was applied to sections for $60 \mathrm{~min}$. Secondary antibody biotinylated goat-antimouse IgG (1:100, Sigma-Aldrich) was applied for $60 \mathrm{~min}$, and streptavidine-conjugated alkaline phosphatase (1:200, Dako). Immunoreactive tissues and cells were stained with an alkaline phosphatase substrate kit (Vector Laboratories, Burlingame, CA). E-cadherin (1:200, Takara, Shiga, Japan) and vimentin (1:50, Dako) immunohistochemistry was performed according to the manufacturer's instructions.

Detection of apoptosis and cell proliferation. To detect apoptotic cells in aggregates, we conducted the terminal deoxynucleotidyl transferase biotin-dUTP nick end-labeling (TUNEL) assay on parallel sections using a commercially available kit (TUNEL apoptosis detection kit, Chemicon, 
Temecula, CA) and the procedure described by the manufacturer. TUNEL staining was performed on sections of paraffin-embedded aggregates (five sections per group, Fig. 6A-C) and on sections of embryos that had been incubated for $48 \mathrm{~h}$ after transplantation (five embryos per group). In the latter case, aggregates that had integrated into the roof plate or remained in the lumen of the neural tube were evaluated (Fig. 6D-F).

To detect DNA synthesis as a marker for cell proliferation, cells were incubated with 5-bromo-2-deoxyuridine (BrdU, Sigma-Aldrich) during aggregate formation. After $22 \mathrm{~h}$ of incubation in roller culture, the biofoil bags were opened, BrdU-solution was added (resulting in $10 \mathrm{ng} / \mathrm{ml}$ ), bags were re-sealed and incubated for another $2 \mathrm{~h}$ in roller culture. Melanoma cell aggregates were fixed with $4 \%$ PFA, embedded in paraffin, and cut into $5-\mu \mathrm{m}$ thick sections. Paraffin sections were pre-treated with $10 \mathrm{mmol} / \mathrm{l}$ citrate buffer $(\mathrm{pH} \mathrm{6.0)}$ ) for $10 \mathrm{~min}$ in a water bath at $90^{\circ} \mathrm{C}$. Anti-BrdU-IgG (1:50, Dako) was applied for $30 \mathrm{~min}$ at room temperature, followed by Cy3 anti-mouse conjugate (1:200, Jackson Laboratories) for $30 \mathrm{~min}$ at room temperature. The sections were imaged using an inverted fluorescence microscope with adequate filter sets and a 20-fold objective. BrdU-positive and negative cells were counted using Analysis 3.0 software (Fig. 5D-F).

Statistical analysis. Statistical analyses of the data generated in this study were performed using Student's t-test and Fisher's exact test (a procedure that calculates the exact probability value between two dichotomous variables for low case numbers, especially if a number $<5$ is comprised in the sample). $\mathrm{P}$-values $<0.05$ were considered statistically significant.

\section{Results}

Treatment with BMP-2 or noggin had no visible effect on the melanoma cell aggregates. A cell suspension of $10^{6} \mathrm{~B} 16-\mathrm{F} 1$ melanoma cells $/ \mathrm{ml}$ was sealed in $1 \mathrm{ml}$ medium in biofoil bags [Fig. 1A, (23)] and kept for $24 \mathrm{~h}$ in rotation culture to form aggregates. The size of the aggregates depended on the geometry of the roller device and the frequency of rotation. The aggregates reached a size of approximately $0.5 \mathrm{~mm}$ in diameter (Fig. 1B). During rotation, BMP-2 or noggin was added. There was no detectable difference in size or shape between BMP-2- or noggin-treated and untreated aggregates. The aggregates resembled neurospheres formed from SVZ cells in non-coated tissue culture dishes $(7,25)$. In paraffin sections of the aggregates, all melanoma cells exhibited HMB-45 immunoreactivity (Figs. 1C and 5A-C).

Cell viability was not affected by treatment. DNA synthesis as an indicator for cell proliferation was measured in one experiment by 5-bromo-2-deoxyuridine (BrdU)-uptake of the melanoma cell aggregates. There was no significant difference between aggregates of all three groups. Approximately $35 \%$ of the cells stained positive for anti-BrdU (Fig. 5D-F). In order to exclude an influence of BMP-2 or noggin on the viability of the melanoma cells, we conducted the terminal deoxynucleotidyl transferase biotin-dUTP nick end-labeling (TUNEL) assay in a second experiment to detect apoptosis. Only a few TUNEL-positive apoptotic melanoma cells were
Table I. List of experiments.

\begin{tabular}{lccc}
\hline Experiment & Treatment & $\mathrm{n}$ & Emigration/no emigration \\
\hline $1(24 \mathrm{~h})$ & Untreated & 4 & $2 / 2$ \\
& BMP-2 & 4 & $3 / 1$ \\
$2(48 \mathrm{~h})$ & Untreated & 4 & $3 / 1$ \\
& BMP-2 & 5 & $5 / 0$ \\
& Noggin & 4 & $1 / 3$ \\
$3(24 \mathrm{~h})$ & Untreated & 4 & $4 / 0$ \\
& BMP-2 & 4 & $4 / 0$ \\
& Noggin & 5 & $0 / 5$ \\
$4(24 \mathrm{~h})$ & BMP-2 & 1 & $0 / 1$ \\
& Noggin & 11 & $0 / 11$ \\
Total & & 46 & $22 / 24$ \\
\hline
\end{tabular}

$\mathrm{n}$, number of embryos. Melanoma cell aggregates were treated with noggin or BMP-2. After transplantation into the neural tube of the chick embryo, emigration of single melanoma cells along the medial neural crest pathway was classified as emigration or no emigration, based on live fluorescence observations and immunohistochemistry after 24 or $48 \mathrm{~h}$ of further incubation.

observed in the three groups of aggregates (Fig. 6). Taken together, pre-treatment with either BMP-2 or noggin did not influence cell proliferation or apoptosis of the melanoma cell aggregates. This excludes an influence on cell viability relevant for the present experiment.

Migration of the B16-F1 melanoma cells was followed by live fluorescence and HMB-45 immunohistochemistry. Untreated, BMP-2- or noggin-treated aggregates were transplanted into the neural tube of the 2-day chick embryo at the location of the 16th-19th pair of somites (corresponding to the prospective site of the upper limb bud; Fig. 4). Four experiments with a total of 46 embryos were performed (Table I). Each experiment started from the same homogeneous cell suspension so that the only difference between aggregates was the treatment. The overall survival rate of transplanted embryos was 46 out of 49 .

Two hours after transplantation, the locations of the transplants were documented by live fluorescence. The aggregates appeared as fluorescent compact rods of cells in the lumen of the neural tubes. The roof plate was intact and the embryos were not damaged except for the caudal spot of penetration with the micro-pipette. At $2 \mathrm{~h}$ no difference in live fluorescence was present between the three groups. After 24 or $48 \mathrm{~h}$ of further incubation, the embryos were excised with intact circulation from the fenestrated eggs and transferred to a temperature-controlled chamber under the inverted fluorescence microscope. After $24 \mathrm{~h}$, emigration of single fluorescent cells from the neural tube was visible in the untreated and BMP-2treated groups but not in the noggin-treated group. After $48 \mathrm{~h}$, emigration of cell clusters was observed in the untreated and BMP-2-treated groups but not after noggin treatment. 

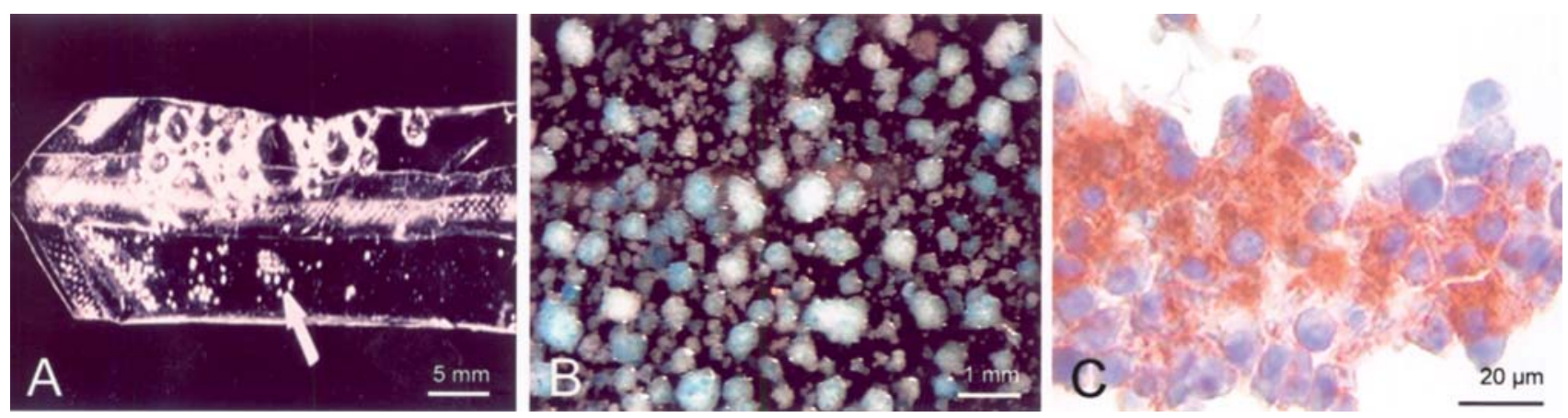

Figure 1. Melanoma cell aggregates used for transplantation. (A) Melanoma cell aggregates (arrow) in a biofoil bag after $24 \mathrm{~h}$ of aggregation time. (B) Aggregates before transplantation, stained with nile blue sulphate for better visibility. (C) Paraffin section of an untreated aggregate, HMB-45 staining. All cells show a positive staining reaction.

\section{4 hours after transplantation}

GFP-epifluorescence
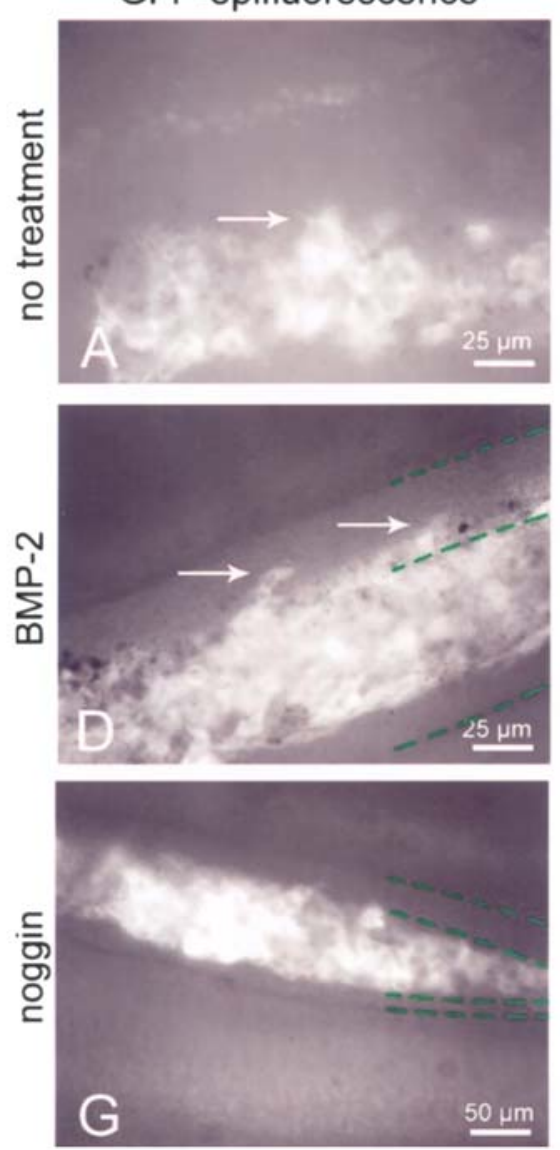
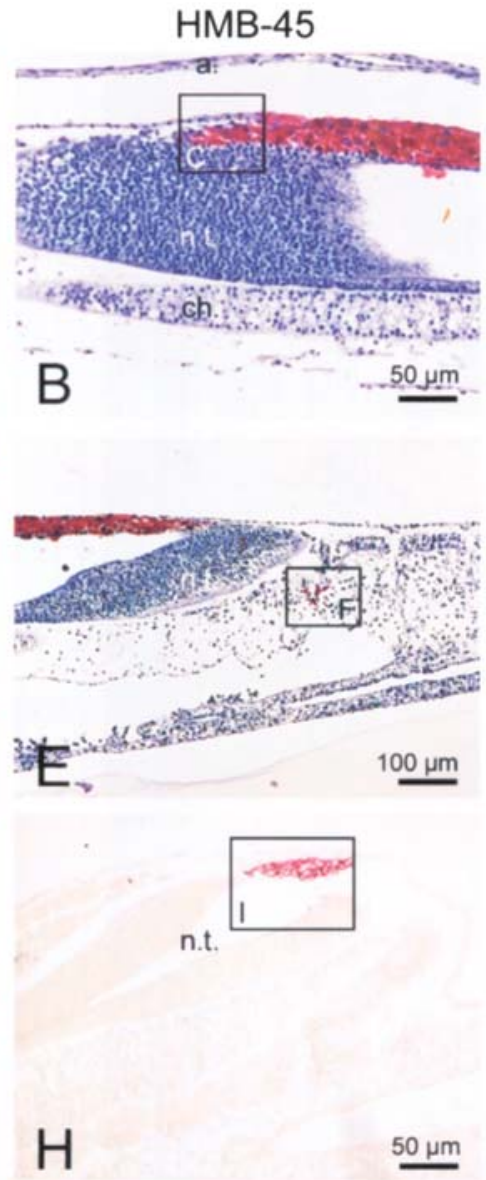

HMB-45
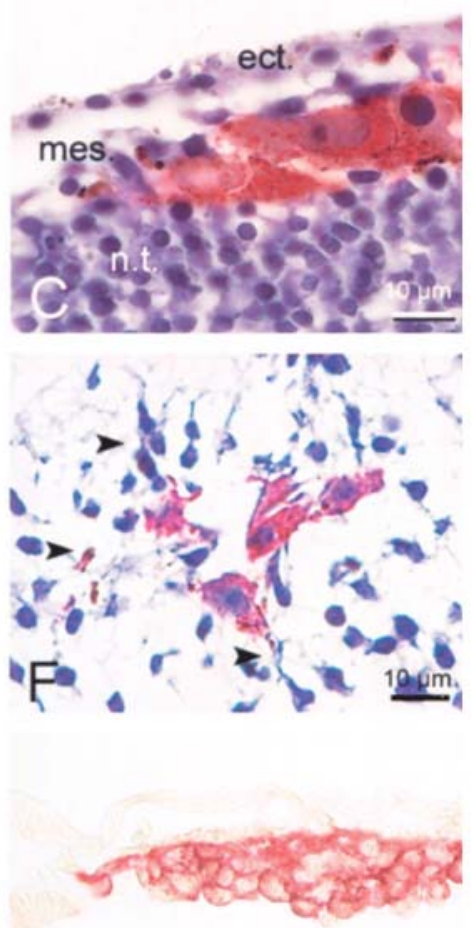

$\underline{25 \mu m}$

Figure 2. Twenty-four hours after transplantation, melanoma cells emigrated from untreated and BMP-2-treated, but not from noggin-treated aggregates. (A-C) Embryo transplanted with an untreated aggregate (A, magnified aggregate with single protruding B16 cells with pseudopodia, arrow). The borders of the neural tube are not discernible due to the plane of focus (compare to G). Immunostaining with HMB-45 (same embryo, B and C) shows the aggregate that has integrated into the roof plate, and single emigrating cells with a stretched morphology. Frame in B indicates higher magnification displayed in C. (D-F) Embryo transplanted with BMP-2-treated aggregate. The borders of the neural tube are indicated by a dotted green line. Fluorescence visualizes B16-F1 cells (D, arrows) migrating from the lumen of the neural tube thus concealing the medial borders of the neural tube (compare to G). The lateral borders of the neural tube are clearly visible. Immunostaining with HMB-45 (same embryo, E and F) displays the aggregate in the roof plate and a group of emigrating melanoma cells with pseudopodia (arrowheads) in the sclerotome. (G-I) Example of an embryo transplanted with a noggin-treated aggregate. GFPfluorescence and the addition of low transmitted light revealed the clearly discernible borders of the neural tube (indicated by dotted green lines) and no emigrating B16-F1 cells (G). Section of the same embryo, immunostaining with HMB-45 (H and I) visualizes the aggregate in the roof plate. The cells exhibit a compact morphology without signs of emigration. n.t., neural tube; ch., chorda dorsalis; a., amnion; ect., surface ectoderm; mes., mesoderm.

For histological evaluation, the same embryos were embedded in paraffin and serially sectioned. In the untreated and BMP-2-treated groups, as expected, the observations of the previous study with B16-F1 mouse melanoma cells (5) 


\section{8 hours after transplantation}

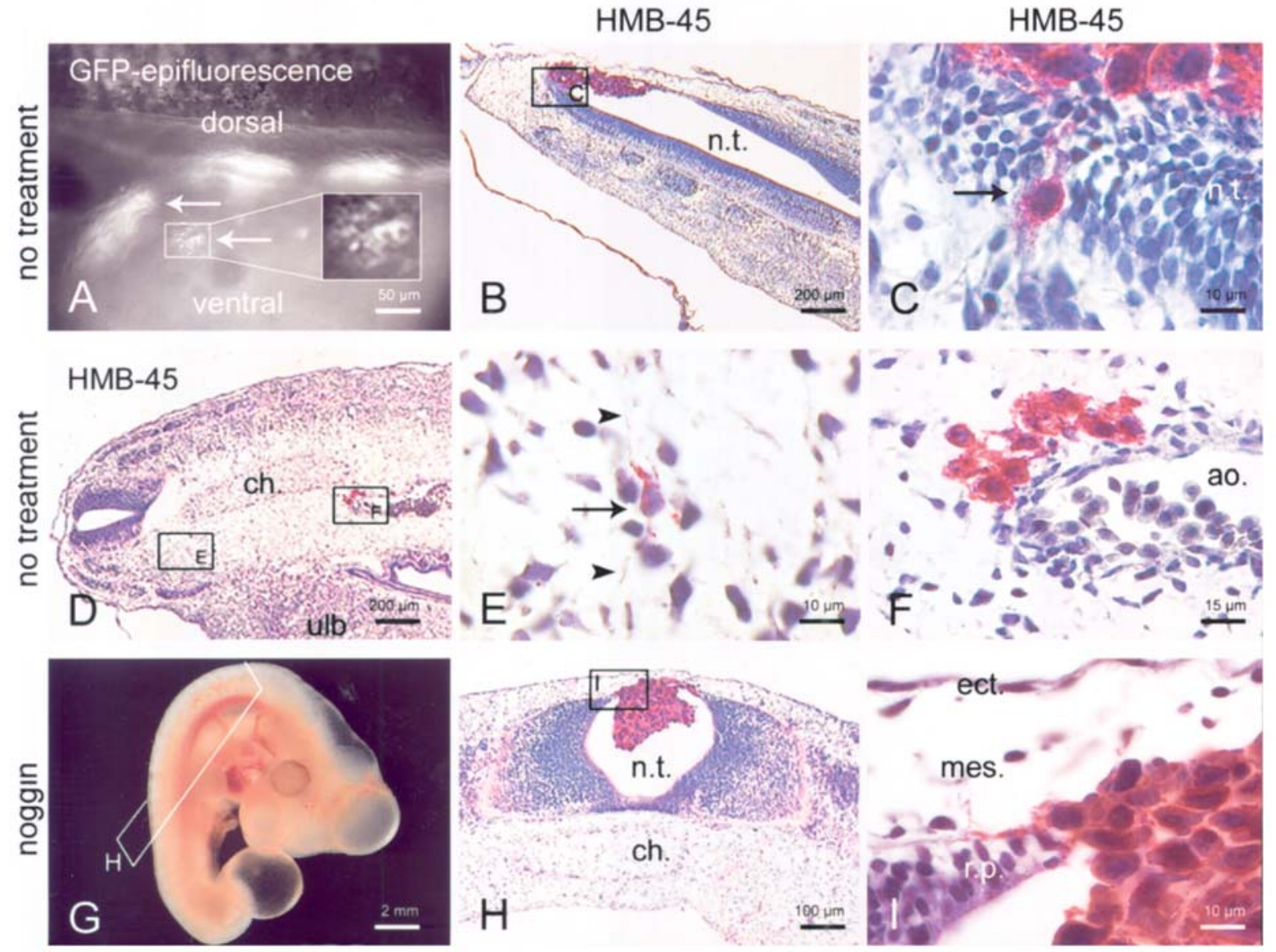

Figure 3. Forty-eight hours after transplantation untreated and BMP-2-treated cells have reached the aorta, while noggin-treated aggregates are still confined to the neural tube. (A) Example of an embryo that was transplanted with an untreated aggregate. Deep ventral emigration of clusters of transplanted melanoma cells (arrows) is visualized by GFP-epifluorescence. Insert demonstrates cellular GFP-epifluorescence. Immunostaining with HMB-45 (B) gives an overview of the same sectioned embryo as in A. Emigration still occurs at this point of time. One B16-F1 melanoma cell is depicted (C) migrating through the roof plate (arrow). The cell extends pseudopodia from the lumen of the neural tube to the mesenchyme and shows a different morphology compared to compact nonemigrating cells remaining in the lumen of the neural tube. (D-F) A different embryo with an untreated aggregate depicting melanoma cells that have emigrated along the medial neural crest pathway. B16-F1 cells (E, arrow) with pseudopodia (E, arrowheads) are found in the sclerotome, and in sympathetic ganglia at the aorta with a more compact morphology (F). (G) Example of a 4-day chick embryo indicating a plane of the section in $\mathrm{H}$. (H and I) Embryo that was transplanted with a noggin-treated aggregate. HMB-45 immunostaining visualizes the aggregate intercalated into the roof plate (H). Higher magnification shows no intermingling of B16-F1 cells with chick mesodermal cells or emigration of B16-F1 cells (I). Melanoma cell morphology is compact with no pseudopodia. n.t., neural tube; ch., chorda dorsalis; ao., aorta; ect., surface ectoderm; mes., mesoderm; r.p., roof plate.

were confirmed. In the present study the cutting direction was in a frontal plane (longitudinal sections, parallel to the aggregate in the neural tube). This cutting direction allowed the depiction of the chain of somites and the para-aortic region with the sympathetic ganglia within the same section. For identification of emigrated melanoma cells, HMB-45 was used on parallel slides. By following this procedure, a thorough screening of each embryo was possible and nonemigration or emigration of melanoma cells was detected reliably in all embryos (Table I).

The complete documentation can be viewed by using the following web-link: www.anatomie.uni-tuebingen.de/anatue/ forsch/melanom. In Table III each embryo is listed individually with live fluorescence observations and histology. Images are attached via a hyperlink which opens a PDF document containing GFP-fluorescence images at 2 and 24 or $48 \mathrm{~h}$ after transplantation and the relevant microscopical sections with HMB-45 immunostaining of the same embryos. In the following section the observations are described in detail.
Emigration occurred from untreated and BMP-2-treated but not from noggin-treated aggregates. Fig. 2 shows live fluorescence and immunohistochemistry of the same embryos of all three groups after $24 \mathrm{~h}$. Fig. 2A, D, and G depicts live fluorescence images of the three groups. Under the inverted fluorescence microscope the projection of the outer and inner borderline of the neuroepithelium and the lumen of the neural tube in-between was visualized by addition of low transmitted light (Fig. 2D and G). Emigrating cells located outside of the projection of the lumen of the neural tube were characterized by fluorescent pseudopodia. Migrating fluorescent cells were only observed in the uppermost layers beneath the amnion and surface ectoderm, and in the roof plate. In Fig. 2A emigrating cells with fluorescent pseudopodia are depicted in the uppermost layer of the aggregate. In Fig. 2D similar migrating cells were observed outside of the lumen above the wall of the neural tube.

In the noggin-treated aggregates (Fig. 2G) the fluorescent melanoma cells were still confined to the lumen of the neural 


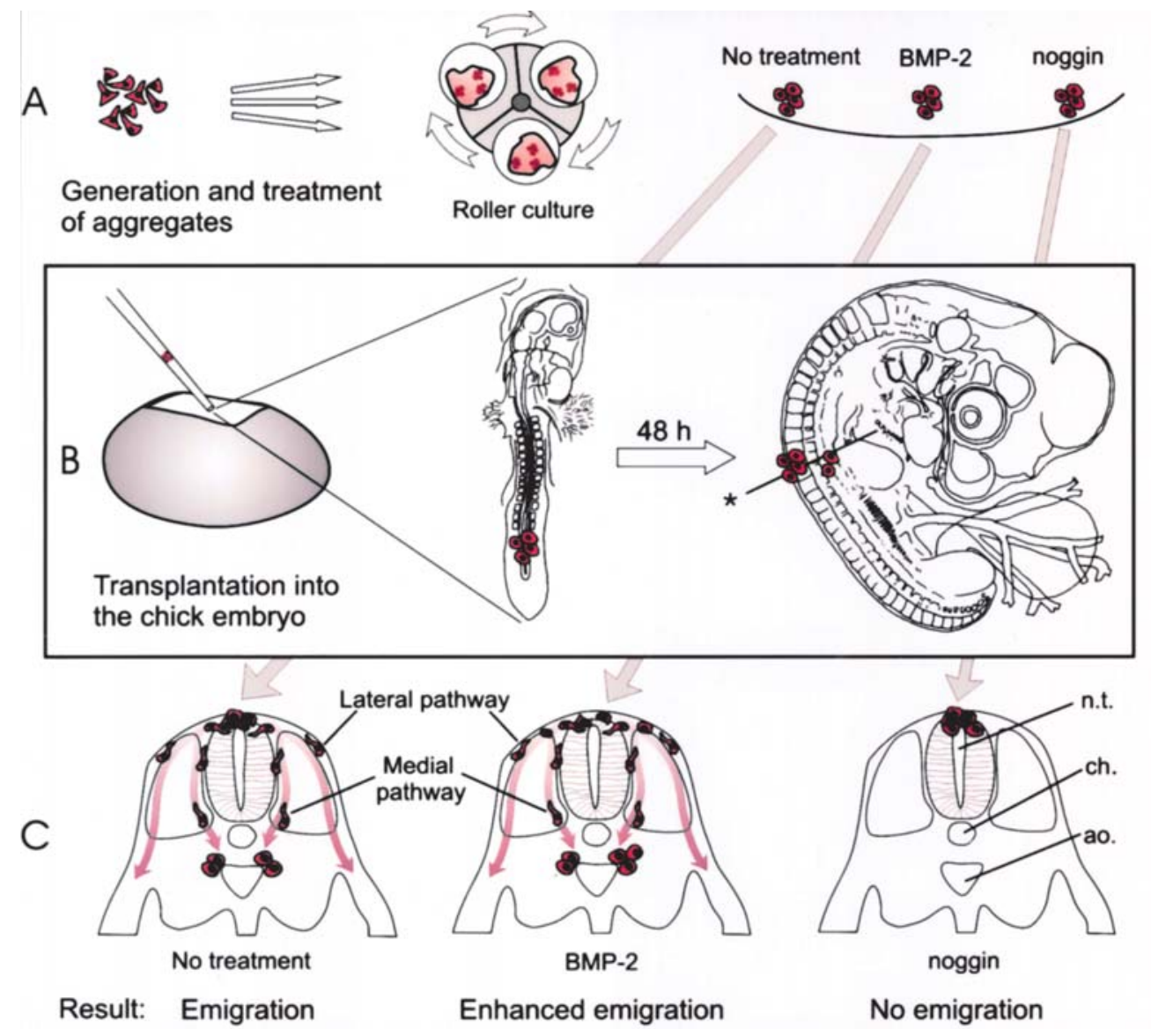

Figure 4. Experimental design. (A) Schematic display of the experimental design and outcome. B16-F1 melanoma cells were incubated in roller culture for $24 \mathrm{~h}$ for the generation of aggregates and treated with BMP-2 or noggin during aggregation in biofoil bags. (B) Aggregates were transplanted into the E2 chick embryo neural tube. (C) After 24 or $48 \mathrm{~h}$ of further incubation, histological slides of the embryos (plane of section indicated by asterisk) were evaluated for neural crest cell-like emigration of the transplanted B16-F1 melanoma cells.
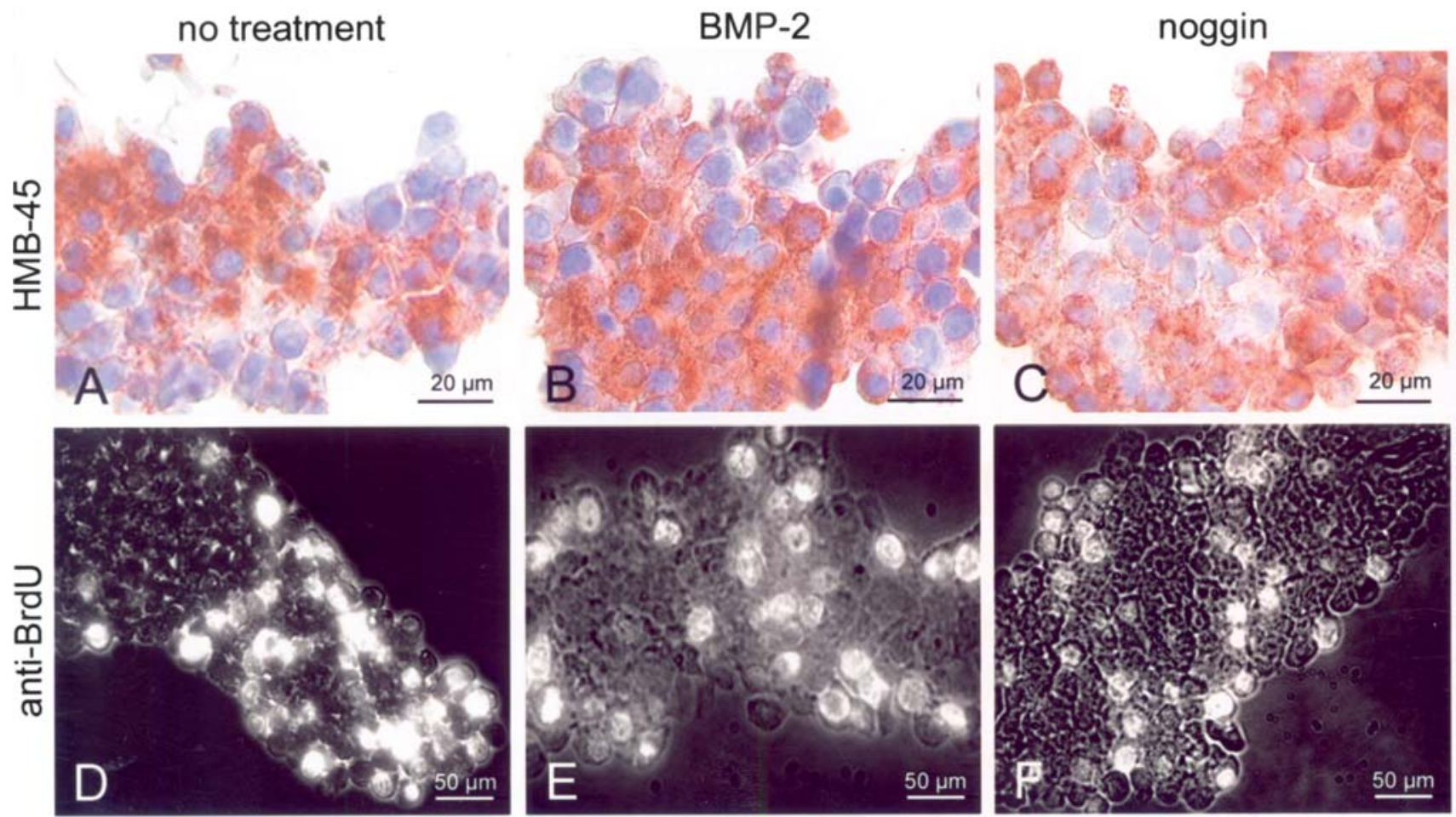

Figure 5. (A-C) Paraffin sections of aggregates, HMB-45 staining. All aggregate cells show a positive staining reaction. No difference in staining pattern is visible between the three groups of aggregates. (D-F) BrdU incorporation in histological sections of B16-F1 aggregates, visualized by anti-BrdU staining. Equal distribution of positively stained melanoma cells indicates no difference in cell proliferation between the three groups. 

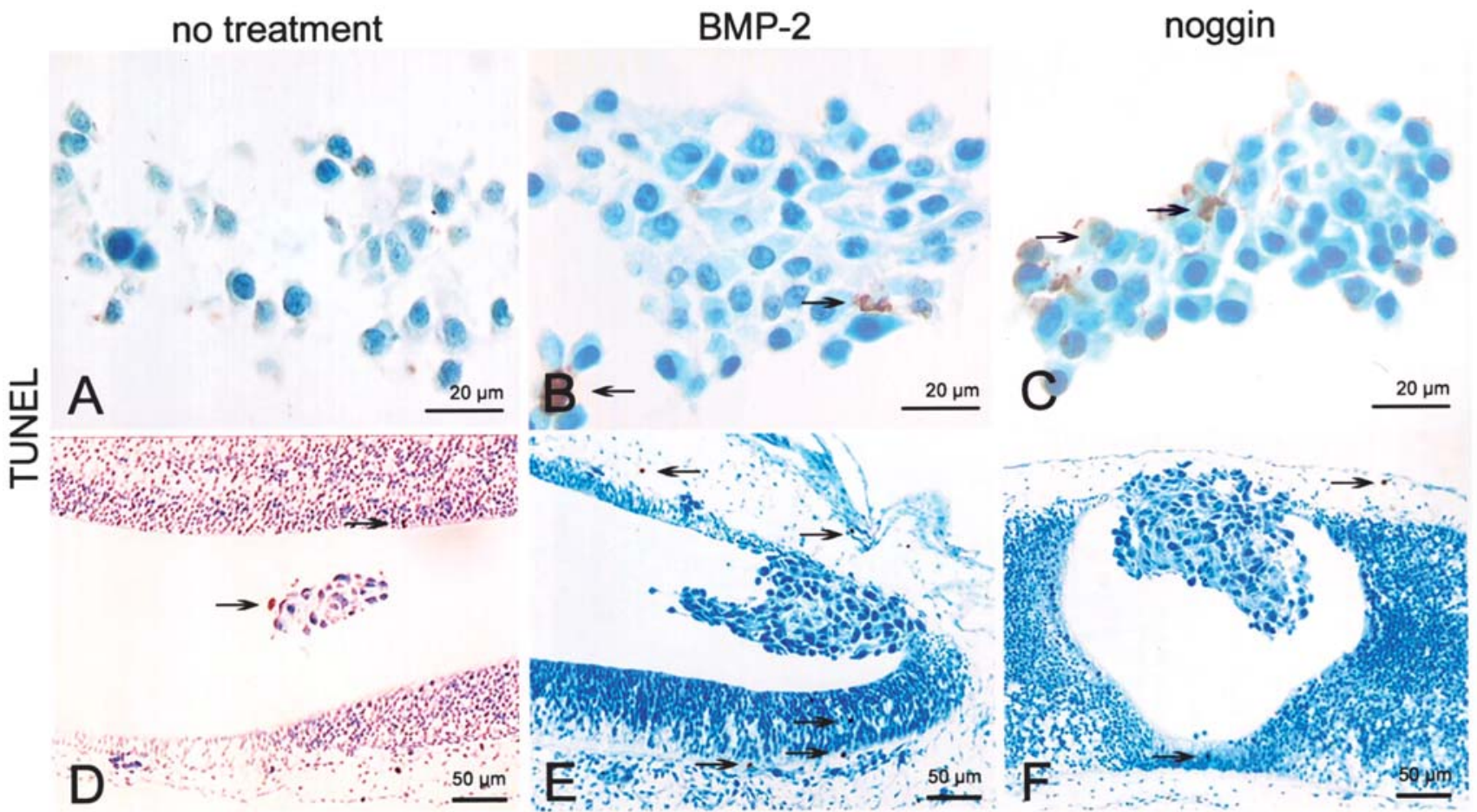

Figure 6. Tunel reaction of aggregates before (A-C) and after (D-F) transplantation. No significant difference in the number of apoptotic cells is visible between untreated (A and D), BMP-2-treated (B and E), and noggin-treated (C and F) aggregates indicating that treatment does not affect cell survival before and after transplantation. Arrows indicate single apoptotic cells detected.

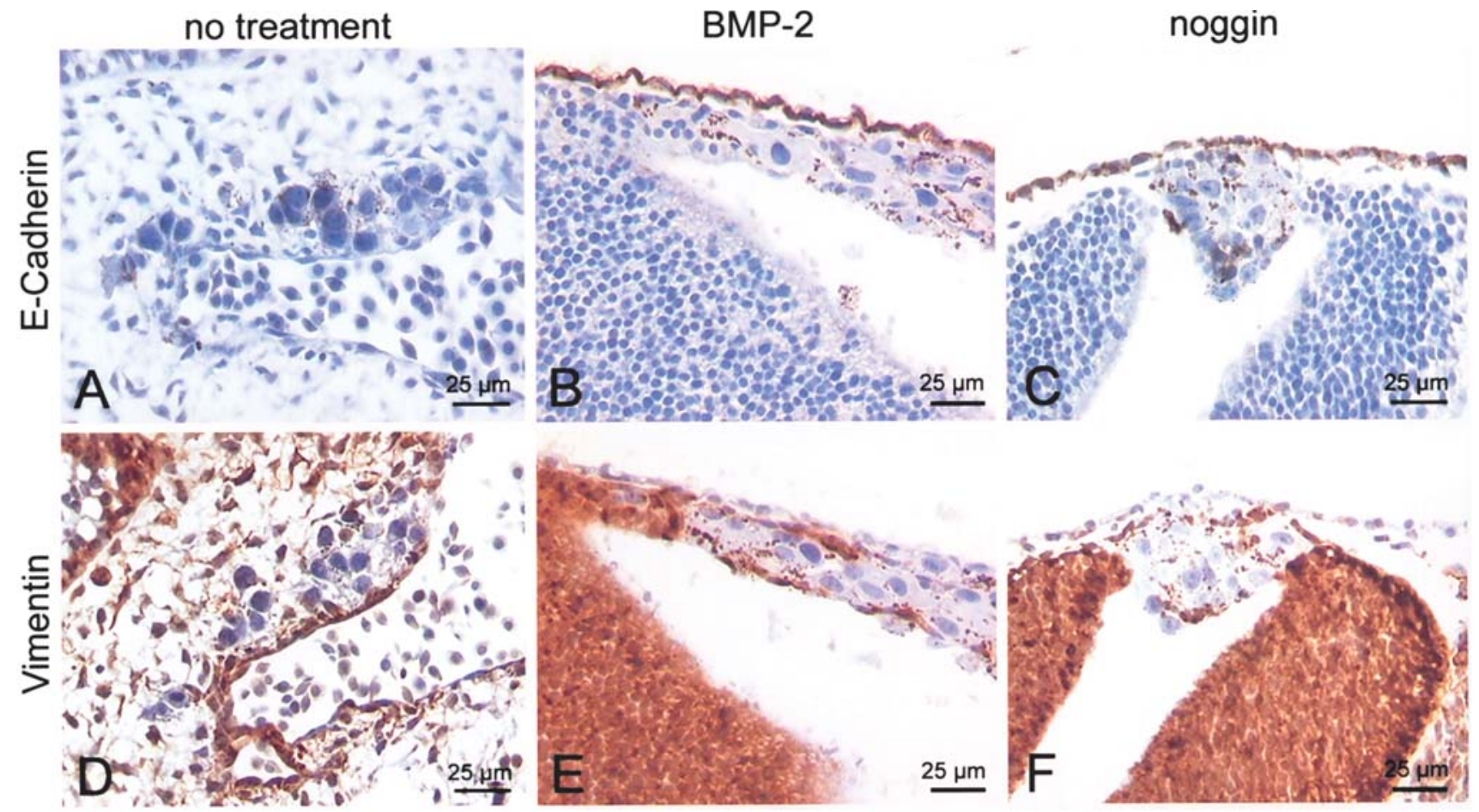

Figure 7. E-cadherin (A-C) and vimentin (D-F) staining of chick embryos $24 \mathrm{~h}$ (B,C,E and F) and $48 \mathrm{~h}$ (A and D) after transplantation of treated and untreated aggregates. Melanoma cells are E-cadherin and vimentin negative independent of treatment or emigration along the neural crest of the chick host.

tube. The vital appearance of noggin-treated aggregates in vivo visualized by GFP-fluorescence was later confirmed by TUNEL staining (Fig. 6).

After $24 \mathrm{~h}$ the embryos had reached stage 18. Histology showed that the roof plate formed a low columnar epithelium with a basal lamina directed towards the mesenchymal layer. The melanoma cell aggregates were intercalated into the roof plate, while the border between chick epithelial cells and HMB-45 positive mouse melanoma cells was clearly visible (Fig. 2B,E and H). The melanoma cells formed a dense 
irregular mass without basal lamina. Melanoma cells from untreated aggregates had begun to emigrate from the roof plate and were detected in the mesenchyme between the neural epithelium and surface ectoderm (Fig. 2B). A higher magnification of the emigrating melanoma cells revealed that the cells possessed a stretched morphology with extended pseudopodia (Fig. 2C) indicating active migration. In the BMP-2 treated aggregate emigrating melanoma cells were detected in the mesenchyme adjacent to the roof plate (Fig. 2E). In addition, stretched cells with pseudopodia were found along the medial neural crest pathway in the sclerotome lateral of the neural tube (Fig. 2E and F).

Noggin-treated aggregates were intercalated as a compact cell mass into the roof plate (Fig. 2H). No HMB-45-positive melanoma cells were encountered outside of the aggregates or along the neural crest pathways. Higher magnification showed that the melanoma cells did not intermingle with mesenchymal host cells and possessed a compact morphology with no pseudopodial extensions beyond the aggregate (Fig. 2I).

In one embryo, blisters formed inside the aggregate (Exp. 2, embryo no. 5, Table III). In contrast to the other noggintreated aggregates, an open border had developed towards the mesodermal layer, and one melanoma cell was observed in one section amidst the chick mesodermal cells. This embryo was rated positive for emigration ( 1 of 20, Table I).

Fig. 3 depicts the observations $48 \mathrm{~h}$ after transplantation. The second time-point confirmed the fluorescence observations made after 24 h. During development, the chick embryo bends and rotates allowing a lateral view of emigrating cells visualized by GFP-epifluorescence. In untreated and BMP-2treated aggregates deep ventral emigration of GFP-positive melanoma cell clusters was detected. Fig. 3A depicts an embryo with an untreated aggregate in the region of the upper limb bud. In the neural tube the fluorescent aggregate is still visible as two horizontally stretched fluorescent nodules. Emigrating clusters of melanoma cells are indicated by arrows.

Histology confirmed that continued emigration of melanoma cells occurred. Fig. 3B shows a longitudinal section of the embryo depicted in Fig. 3A. One melanoma cell is displayed in higher magnification migrating through the roof plate, stretching pseudopodia from the lumen of the neural tube to the mesenchyme (Fig. 3C). Melanoma cells were observed along the whole medial neural crest pathway through the sclerotome to the sympathetic chain. In the sclerotome, cells with extensive pseudopodia are depicted (Fig. 3E). Interestingly, melanoma cells that had reached the sympathetic chain had a rather compact morphology with only a few pseudopodia (Fig. 3F).

Noggin-treated aggregates remained in the lumen of the neural tube as a compact mass of cells or were intercalated into the roof plate without cellular emigration (Fig. $3 \mathrm{H}$ ). Higher magnification showed that the cells still possessed their compact morphology and did not intermingle with the surrounding chick mesenchymal cells (Fig. 3I).

BMP-2 treatment enhanced emigration of melanoma cells. Emigration of BMP-2-treated aggregates was qualitatively enhanced compared to untreated aggregates with regard to the number of emigrating cells and the distance reached. In 2 (both after $48 \mathrm{~h}$ ) of the 9 embryos with untreated aggregates
Table II. Statistical evaluation of experiments.

\begin{tabular}{lccl}
\hline Treatment & $\mathrm{n}$ & Emigration/no emigration & $\mathrm{p}$-value \\
\hline Untreated & 12 & $9 / 3$ & 1 \\
BMP-2 & 14 & $12 / 2$ & 0.347 \\
Noggin & 20 & $1 / 19$ & $0.000069^{a}$ \\
\hline
\end{tabular}

n, number of embryos; p, probability, Fisher's exact test, two-tailed. After transplantation, emigration of BMP-2- or noggin-treated melanoma cells was compared to emigration of untreated melanoma cells with Fisher's exact test. p-values $<0.05$ were considered as a significant difference. Although an enhanced emigration in the single embryo was observed after additional BMP-2 treatment, the number of embryos with emigration (12 of 14) as compared to emigration of the number of embryos with untreated aggregates (9

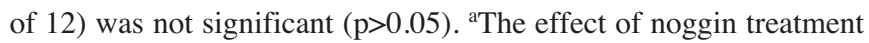
was highly significant $(\mathrm{p}<0.0001)$.

in which local emigration in the area of the roof plate and the mesenchyme had occurred, also emigration of melanoma cells along the medial neural crest pathway in the area of the para-aortic ganglia was detected. The same phenomenon was observed in 7 ( 4 after $24 \mathrm{~h}$ and 3 after $48 \mathrm{~h}$ ) of the 12 embryos with BMP-2-treated aggregates and local emigration.

Statistical evaluation. For evaluation of the BMP-2 and noggin effects (Table I), the criterion for emigration and no emigration was the presence of HMB-45-positive cells in the mesenchymal layer between the surface ectoderm and the roof plate. Embryos with single HMB-45-positive melanoma cells emigrating from the neural tube, intermingling with chick host cells in the mesoderm dorsally and laterally of the roof plate, or along the medial neural crest pathway in the region of the aorta were rated as 'emigration' (Figs. 2C and F; and $3 \mathrm{C}$ and $\mathrm{F}$ ). When no melanoma cells had left the compound structure of the aggregate in the roof plate, the transplanted embryo was rated as 'no emigration' (Figs. 2I and 3I). Based on these counts, levels of significance were calculated with Fisher's exact test. Noggin suppressed melanoma cell emigration in 19 out of 20 embryos. Statistically this inhibition of emigration was highly significant in comparison with untreated aggregates $(\mathrm{p}<0.0001$, Table II). The enhancing effect of BMP-2 on melanoma cell emigration was not statistically significant $(\mathrm{p}>0.05)$.

Melanoma cells did not stain for E-cadherin or vimentin. In order to further characterize the B16-F1 cells during their neural crest cell-like migration, we performed E-cadherin and vimentin immunohistochemistry on parallel slides to test whether the transplanted cells exhibited epithelial [E-cadherin (26)] or mesenchymal [vimentin (27)] traits. B16-F1 cell aggregates were negative for both E-cadherin and vimentin in the treated and the untreated groups before transplantation (not shown) and after $48 \mathrm{~h}$ of incubation after transplantation (Fig. 7). In the chick host embryo E-cadherin was expressed in the surface ectoderm and vimentin in the neuroepithelium of the neural tube. 
Table III. An overview of all 46 embryos included in this study.

Experiment Noggin BMP-2 Control Incubation time (p.t.) Fluorescence of emigration (+/-) Histology of emigration (+/-)

\begin{tabular}{|c|c|c|c|c|c|c|}
\hline \multirow[t]{8}{*}{1} & & & E2 & $24 \mathrm{~h}$ & $(-)$ & $+($ mesodermal $)$ \\
\hline & & & E3 & $24 \mathrm{~h}$ & $(-)$ & - \\
\hline & & & $\mathrm{E} 4$ & $24 \mathrm{~h}$ & $(-)$ & $+($ mesodermal $)$ \\
\hline & & & E5 & $24 \mathrm{~h}$ & $(-)$ & - \\
\hline & & E6 & & $24 \mathrm{~h}$ & $(-)$ & $\begin{array}{l}+ \text { (mesodermal, para-aortic } \\
\text { ganglia) }\end{array}$ \\
\hline & & E7 & & $24 \mathrm{~h}$ & $(-)$ & $+($ mesodermal $)$ \\
\hline & & E8 & & $24 \mathrm{~h}$ & $(-)$ & - \\
\hline & & E9 & & $24 \mathrm{~h}$ & + & $+($ mesodermal $)$ \\
\hline \multirow[t]{13}{*}{2} & & & E1 & $48 \mathrm{~h}$ & + & $+($ para-aortic ganglia $)$ \\
\hline & & & E2 & $48 \mathrm{~h}$ & + & + (para-aortic ganglia) \\
\hline & & & E3 & $48 \mathrm{~h}$ & - & - \\
\hline & $\mathrm{E} 4$ & & & $48 \mathrm{~h}$ & - & - \\
\hline & E5 & & & $48 \mathrm{~h}$ & - & + (blister) \\
\hline & & E7 & & $48 \mathrm{~h}$ & + & $+($ dorsally of aorta $)$ \\
\hline & & E8 & & $48 \mathrm{~h}$ & $+/-$ & $+(2$ cells in mesoderm $)$ \\
\hline & & E9 & & $48 \mathrm{~h}$ & $+/-$ & $+($ dorsally of aorta $)$ \\
\hline & & E10 & & $48 \mathrm{~h}$ & + & + (dorsally of aorta) \\
\hline & E11 & & & $48 \mathrm{~h}$ & - & - \\
\hline & E12 & & & $48 \mathrm{~h}$ & - & - \\
\hline & & & E13 & $48 \mathrm{~h}$ & + & $+($ mesodermal $)$ \\
\hline & & E14 & & $48 \mathrm{~h}$ & + & $+($ mesodermal $)$ \\
\hline \multirow[t]{13}{*}{3} & E1 & & & $24 \mathrm{~h}$ & - & - \\
\hline & E2 & & & $24 \mathrm{~h}$ & - & - \\
\hline & E3 & & & $24 \mathrm{~h}$ & $(-)$ & - \\
\hline & $\mathrm{E} 4$ & & & $24 \mathrm{~h}$ & - & - \\
\hline & & E5 & & $24 \mathrm{~h}$ & + & $+($ sclerotome $)$ \\
\hline & & E6 & & $24 \mathrm{~h}$ & + & $+($ mesodermal $)$ \\
\hline & & E7 & & $24 \mathrm{~h}$ & + & $+($ sclerotome $)$ \\
\hline & & E8 & & $24 \mathrm{~h}$ & + & $+($ mesodermal $/$ sclerotome $)$ \\
\hline & & & E9 & $24 \mathrm{~h}$ & + & $+($ mesodermal $)$ \\
\hline & & & E10 & $24 \mathrm{~h}$ & + & $+($ mesodermal $)$ \\
\hline & & & E11 & $24 \mathrm{~h}$ & $+/-$ & $+($ mesodermal $)$ \\
\hline & & & E12 & $24 \mathrm{~h}$ & $+/-$ & $+($ mesodermal $)$ \\
\hline & E13 & & & $24 \mathrm{~h}$ & - & - \\
\hline \multirow[t]{12}{*}{4} & E1 & & & $24 \mathrm{~h}$ & - & - \\
\hline & E2 & & & $24 \mathrm{~h}$ & - & - \\
\hline & E3 & & & $24 \mathrm{~h}$ & - & - \\
\hline & $\mathrm{E} 4$ & & & $24 \mathrm{~h}$ & - & - \\
\hline & E5 & & & $24 \mathrm{~h}$ & - & - \\
\hline & E7 & & & $24 \mathrm{~h}$ & - & - \\
\hline & E8 & & & $24 \mathrm{~h}$ & - & - \\
\hline & E9 & & & $24 \mathrm{~h}$ & - & - \\
\hline & E10 & & & $24 \mathrm{~h}$ & - & - \\
\hline & E11 & & & $24 \mathrm{~h}$ & - & - \\
\hline & E12 & & & $24 \mathrm{~h}$ & $+/-$ & \\
\hline & & E13 & & $24 \mathrm{~h}$ & + & \\
\hline Total & 20 & 14 & 12 & 24 h: $33 ; 48$ h: 13 & & \\
\hline
\end{tabular}

A detailed overview of all embryos evaluated for this study. Additional live fluorescence and histological images can be viewed by hyperlink at www.anatomie.uni-teubingen.de/anatue/forsch/melanom. 


\section{Discussion}

It has long been proposed that malignant growth is caused by an untimely reappearance of embryonic proliferation and cellular motility in the stem cell compartments of adult organs with regenerative capacity $(1,3)$. The experimental approach of transplanting malignant sarcoma 180 cells into the neural crest of the chick embryo (beneath the ectoderm between the neural tube and the last few caudal somites, so that the transplant lies in the neural crest migratory space) was performed previously (28). Here the regulation of the migratory behaviour of malignant mouse melanoma cells was analyzed in a similar chick embryo transplantation model (transplantation into the neural tube of the chick embryo). The results show that the neural crest cell migration of the melanoma cells described in earlier studies $(4,5)$ is totally inhibited by the BMP-antagonist noggin. The experimental procedure and the results are summarized in Fig. 4: From a melanoma cell suspension (Fig. 4A) small aggregates were prepared and treated with BMP-2 or its antagonist noggin. After transplantation (Fig. 4B) into the embryonic neural tube of the 2-day chick, major differences in the morphogenetic behaviour of the melanoma cells became visible (Fig. 4C). From non-treated aggregates melanoma cells emigrated as expected together with the autochthonous chick neural crest cells. Emigration was totally blocked in noggin-treated aggregates. After BMP-2 treatment emigration was slightly enhanced. The noggin effect was reproduced in 19 out of 20 embryos and was statistically significant when compared to embryos with untreated aggregates.

In this study we have shown that noggin treatment of melanoma cells abrogates their inherent potential to emigrate from the embryonic neural crest of a chick host embryo. Thus, migration of malignant melanoma cells can be influenced by the embryonic regulatory molecule noggin.

BMP is an inductor of neural crest cell migration, whereas noggin acts as an inhibitor $(15,16)$. Neural crest cell migration of non-malignant neural stem cells is dependent on pretreatment with BMP-2, while malignant melanoma cells migrate without exogenous BMP due to their constitutive BMP expression. Neural stem cells from the subventricular zone (SVZ) of adult mice transplanted into the chick embryo neural tube integrated into the neural crest only after exogenous BMP-2 pre-treatment (13). In contrast, transplanted human SK-Mel 28 (4) and mouse B16-F1 (5) melanoma cells integrated into the neural crest without BMP-2 treatment. The explanation for these observations is the constitutive BMP expression observed in 9 human melanoma cell lines by Rothhammer et al (18). The noggin effect described here demonstrates that the neural crest migration of melanoma cells in the chick embryo is driven by endogenous BMP. The BMP-noggin antagonism regulated not only neural crest cell emigration from the neural tube; experimental overexpression of noggin in the gut of the chick embryo inhibits normal migration of enteric neural crest cells (29).

Constitutive expression of BMP is not restricted to malignant melanoma cells and can be considered a common phenomenon in malignant cells. It has been shown that BMP mRNAs are expressed in a variety of human carcinoma cell lines, e.g. osteosarcoma (30), salivary adenocarcinoma (31), prostate (32), pancreas (33), ovarian (34), and breast (35).
Moreover, BMP-2 stimulates angiogenesis in developing tumours formed from A549 lung cancer cells injected into athymic nude mice (36). It would be worth studying a possible effect of noggin also in respective cell lines.

BMP-2 and noggin influenced the morphogenesis of the B16-F1 melanoma cells but not their state of determination. In the human SK-Mel 28 and the mouse B16 melanoma cells, melanin synthesis is arrested at the pre-melanosome stage. The preservation of the pre-melanosome epitope HMB-45 after transplantation into the neural tube of the two-day chick embryo indicates that the melanoma cells did not change the state of differentiation. In the host chick embryo this stage of determination is reached after 4 days of incubation. The melanocyte lineage is induced in the lateral pathway of the neural crest cell migration by the subectodermal lateral mesoderm and was visualized by MelEM Mab and ab732 immunoreactivity (37). Despite the determination as melanocytes, the melanoma cells participate in migration of neural crest cells at an earlier state of determination. In addition to the lateral pathway destined for the melanocytic lineage $(4,5)$, they follow the medial pathway leading into the sympathetic ganglia. In this ventral location, they can be detected among host neural crest cells in the para-aortic sympathetic ganglia, where they exhibit no HNK-1 immunoreactivity, as opposed to host neural crest cells (5). Thus, migratory behaviour and state of determination are not correlated. In the embryonic environment the malignant cells were not reprogrammed but rather changed their adhesive and migratory capacities. Reprogramming of human melanoma cells after injection into the neural tube of the chick embryo was reported by others (38). In the latter publication human GFP-expressing (transient cell expression achieved by adenoviral infection) melanoma cells were used. For the detection of the transplanted melanoma cells in the chick host, GFP-epifluorescence and anti-GFP-immunofluorescence were used. It was not excluded that the GFP-protein was passed on to or phagocytosed by host neural crest cells after apoptosis of the transplanted melanoma cells. In contrast to our studies $(4,5)$ the transplanted cells were not unambiguously identified by in situ hybridization with species-specific probes.

Although the state of determination was not changed by BMP-2 or noggin pre-treatment, a permanent change in the migratory potential occurred which involved the capability to participate in epithelial mesenchymal transformation (EMT). In the embryo, emigration of neural crest cells from the neural tube is designated as EMT. EMT represents a complex change in cell morphology and migratory potential of embryonic cells and is induced in the embryo by dorsalizing BMP and inhibited by ventralizing sonic hedgehog and noggin signalling (39). EMT comprises two consecutive steps (40): First, the neural crest compartment is induced in the epithelium of the neural tube. This step is morphologically characterized by the disintegration of the basal lamina in the region of the lateral roof plate. Second, neural crest cells are induced to start migration from the dorsal edges of the neural tube along their designated medial and lateral pathways. Our experiments show that the melanoma cells have the morphological characteristics of neural crest cells after the first step of neural crest induction. After transplantation, they are intercalated in the epithelial roof plate of the neural tube but synthesize no basal 
lamina. The second step, emigration into the mesenchymal surrounding, is BMP and noggin dependent. Noggin treatment of the aggregates probably leads to changes in the adhesive characteristics known to occur during neural crest cell migration. EMT plays a fundamental role in the progression of malignant epithelial tumours, and increased motility and invasiveness of cancer cells have been attributed to processes similar to EMT during embryonic development (41).

Endogenous BMP induces the capability of physiological neural crest cell morphogenesis in melanoma cells with subsequent migration in vivo which can efficiently be antagonized by pre-treatment with noggin. Further experiments confirming and extending the relevance of these observations for the invasive growth of melanoma cells in the optic cup of the chick embryo have been conducted (42).

\section{Acknowledgements}

We would like to thank Ingrid Epple for preparing the paraffin sections and Leokadia Macher for the immunohistochemistry. HMB-45 staining was performed by Susanne Herrgott, Department of Dermatology. E-cadherin and vimentin staining was kindly performed by Dr Manfred Wehrmann, Institute of Pathology, University of Tübingen.

\section{References}

1. Noda S, Lammerding-Köppel M, Oettling G and Drews U: Characterization of muscarinic receptors in the human melanoma cell line SK-Mel-28 via calcium mobilization. Cancer Lett 133: 107-114, 1998.

2. Petty AP, Garman KL, Winn VD, Spidel CM and Lindsey JS: Overexpression of carcinoma and embryonic cytotrophoblast cell-specific mig-7 induces invasion and vessel-like structure formation. Am J Pathol 170: 1763-1780, 2007.

3. Dodelet VC and Pasquale EB: Eph receptors and Ephrin ligands: embryogenesis to tumorigenesis. Oncogene 19: 5614-5619, 2000.

4. Schriek G, Oppitz M, Busch C, Just L and Drews U: Human SK-Mel 28 melanoma cells resume neural crest cell migration after transplantation into the chick embryo. Melanoma Res 15: 225-234, 2005.

5. Oppitz M, Busch C, Schriek G, Just L, Metzger M and Drews U: Non-malignant migration of B16 mouse melanoma cells in the neural crest and invasive growth in the eye cup of the chick embryo. Melanoma Res 17: 17-30, 2007.

6. Le Douarin NM and Kalcheim C: The Neural Crest. 2nd edition. Cambridge University Press, Cambridge, p469, 1999.

7. Taatjes DJ, Arendash-Durand B, von Turkovich D and Trainer TD: HMB-45 antibody demonstrates melanosome specificity by immunoelectron microscopy. Arch Pathol Lab Med 117: 264-268, 1993.

8. Kinsey DL: Hyperbaric oxygen and 5-fluouracil in the treatment of experimental melanoma. Surg Forum 15: 205-206, 1964.

9. Fidler IJ: Selection of successive tumour lines for metastasis. Nature New Biol 242: 148-149, 1973

10. Fidler IJ: Biological behaviour of malignant melanoma cells correlated to their survival in vivo. Cancer Res 35: 218-224, 1975

11. Carl UD, Pollmann M, Orr E, Gertler FB, Chakraborty T and Wehland J: Aromatic and basic residues within the EVH1 domain of VASP specify its interaction with proline-rich ligands. Curr Biol 9: 715-718, 1999.

12. Rottner K, Behrend B, Small JV and Wehland J: VASP dynamics during lamellipodia protrusion. Nature Cell Biol 1: 321-322, 1999.

13. Busch C, Oppitz M, Sailer MH, Just L, Metzger M and Drews U: BMP-2 dependent integration of mouse adult subventricular stem cells into the neural crest of chick and quail embryos. J Cell Sci 119: 4467-4474, 2006.

14. Sailer MH, Hazel TG, Panchision DM, Hoeppner DJ, Schwab ME and McKay RD: BMP2 and FGF2 cooperate to induce neuralcrest-like fates from fetal and adult CNS stem cells. J Cell Sci 118: 5849-5860, 2005 .
15. Sela-Donenfeld D and Kalcheim C: Regulation of the onset of neural crest migration by coordinated activity of BMP4 and noggin in the dorsal neural tube. Development 126: 4749-4762, 1999.

16. Hogan BL: Bone morphogenetic proteins in development. Curr Opin Genet Dev 6: 432-438, 1996.

17. Miyazono $\mathrm{K}$, Ichijo $\mathrm{H}$ and Heldin $\mathrm{CH}$ : Transforming growth factor-beta: latent forms, binding proteins and receptors. Growth Factors 8: 11-22, 1993.

18. Rothhammer T, Poser I, Soncin F, Bataille F, Moser M and Bosserhoff AK: Bone morphogenic proteins are overexpressed in malignant melanoma and promote cell invasion and migration. Cancer Res 65: 448-456, 2005.

19. Zhu W, Kim J, Cheng C, Rawlins BA, Boachie-Adjei O, Crystal RG and Hidaka C: Noggin regulation of bone morphogenetic protein (BMP) 2/7 heterodimer activity in vitro. Bone 39: 61-71, 2006.

20. Zimmermann LB, De Jesus-Escobar JM and Harland RM: The Spemann organizer signal noggin binds and inactivates bone morphogenetic protein 4. Cell 86: 599-606, 1996.

21. Feeley BT, Krenek L, Liu N, Hsu WK, Gamradt SC, Schwarz EM, Huard J and Lieberman JR: Overexpression of noggin inhibits BMP-mediated growth of osteolytic prostate cancer lesions. Bone 38: 154-166, 2006.

22. Chen D, Zhao M and Mundy GR: Bone morphogenetic proteins. Growth Factors 22: 233-241, 2004.

23. Drews U, Ebensperger C and Wolf U: An in vitro model of gonad differentiation in the chick embryo. Roller cultures in gas permeable biofoil bags. Anat Embryol 178: 529-536, 1988.

24. Hamburger V and Hamilton HL: A series of normal stages in the development of the chick embryo (1951). Dev Dyn 195: 231-272, 1992.

25. Bez A, Corsini E, Curti D, Biggiogera M, Colombo A, Nicosia RF, Pagano SF and Parati EA: Neurosphere and neurosphere-forming cells: morphological and ultrastructural characterization. Brain Res 993: 18-29, 2003.

26. Nakagawa $S$ and Takeichi M: Neural crest emigration from the neural tube depends on regulated cadherin expression. Development 125: 2963-2971, 1998.

27. Noack C, Pröls F, Gamel AJ, Rist W, Mayer MP and Brand-Saberi B: Revisiting vimentin expression in early chick development. Anat Embryol 206: 391-397, 2003.

28. Erickson CA, Tosney KW and Weston JA: Analysis of migratory behavior of neural crest and fibroblastic cells in embryonic tissues. Dev Biol 77: 142-156, 1980.

29. Goldstein AM, Brewer KC, Doyle AM, Nagy N and Roberts DJ: BMP signaling is necessary for neural crest cell migration and ganglion formation in the enteric nervous system. Mech Dev 122: 821-833, 2005

30. Gobbi G, Sangiorgi L, Lenzi L, et al: Seven BMPs and all their receptors are simultaneously expressed in osteosarcoma cells. Int J Oncol 20: 143-147, 2002.

31. Hatakeyama S, Ohara-Nemoto Y, Kyakumoto S and Satoh M: Expression of bone morphogenetic protein in human adenocarcinoma cell line. Biochem Biophys Res Commun 190: 695-701, 1993.

32. Ide H, Yoshida T, Matsumoto N, Aoki K, Osada Y, Sugimura T and Terada M: Growth regulation of human prostate cancer cells by bone morphogenetic protein-2. Cancer Res 57: 5022-5027, 1997.

33. Kleeff J, Maruyama H, Ishiwata T, Sawhney H, Friess H, Buchler MW and Korc M: Bone morphogenetic protein 2 exerts diverse effects on cell growth in vitro and is expressed in human pancreatic cancer in vivo. Gastroenterology 116: 1202-1216, 1999.

34. Kiyozuka Y, Nakagawa H, Senzaki H, Uemura Y, Adachi S, Teramoto Y, Matsuyama T, Bessho K and Tsubura A: Bone morphogenetic protein-2 and type IV collagen expression in psammoma body forming ovarian cancer. Anticancer Res 21: 1723-1730, 2001.

35. Arnold SF, Tims E and Mcgrath BE: Identification of bone morphogenetic proteins and their receptors in human breast cancer cell lines: importance of BMP2. Cytokine 11: 1031-1037, 1999.

36. Langenfeld EM and Langenfeld J: Bone morphogenetic protein-2 stimulates angiogenesis in developing tumours. Mol Cancer Res 2: $141-149,2004$.

37. Nataf V, Mercier P, Ziller C and Le Douarin NM: Novel markers of melanocyte differentiation in the avian embryo. Exp Cell Res 207: 171-182, 1993 . 
38. Kulesa PM, Kasemeier-Kulesa JC, Teddy JM, Margaryan NV, Seftor EA, Seftor RE and Hendrix MJ: Reprogramming metastatic melanoma cells to assume a neural crest-like cell-phenotype in an embryonic microenvironment. Proc Natl Acad Sci USA 103: 3752-3757, 2006.

39. Hay ED: The mesenchymal cell, its role in the embryo, and the remarkable signaling mechanisms that create it. Dev Dyn 233: 706-720, 2005 .

40. Newgreen DF and Minichiello J: Control of epitheliomesenchymal transformation. I. Events in the onset of neural crest cell migration are separable and inducible by protein kinase inhibitors. Dev Biol 170: 91-101, 1995.
41. Zhou BP, Deng J, Xia W, Xu J, Li YM, Gunduz M and Hung MC: Dual regulation of Snail by GSK-3beta-mediated phosphorylation in control of epithelial-mesenchymal transition. Nat Cell Biol 6: 931-940, 2004.

42. Busch C, Drews U, Eisele SR, Garbe C and Oppitz M: Noggin blocks invasive growth of murine B16-F1 melanoma cells in the optic cup of the chick embryo. Int J Cancer (In press). 